\title{
MiR-26a-5p Targets WNT5A to Protect Cardiomyocytes from Injury Due to Hypoxia/Reoxygenation Through the Wnt//-catenin Signaling Pathway
}

\author{
Guohui Yan, ${ }^{1,2, *}$ MD, Jiajia Wang, ${ }^{3, *}$ MD, Zanxi Fang, ${ }^{3}$ MD, Shuidi Yan, ${ }^{3}$ MD and Yang Zhang, ${ }^{2,3}$ MD
}

\begin{abstract}
Summary
This study aimed to investigate the effect and mechanism of miR-26a-5p on cardiomyocyte injury induced by hypoxia/reoxygenation (H/R).

After construction of an H/R model in rat cardiomyocyte H9c2 cells, miR-26a-5p in the cells was interfered with (cells transfected with miR-26a-5p inhibitor) or overexpressed (cells transfected with a miR-26a-5p mimics). The viability and the apoptosis rate of cells in each group were detected using CCK-8 and flow cytometry; the relationship between miR-26a-5p and WNT5A was verified by a dual-luciferase reporter assay; the expression of miR-26a-5p, WNT5A, cleavedcaspase 3 and Wnt/ $\beta$-catenin signaling pathway-related proteins in each group was detected using qRT-PCR or Western blot; LDH release, SOD, and GSH-PX activities in each group were detected by kit.

In the $\mathrm{H} / \mathrm{R}$ group, the expression level of miR-26a-5p was significantly decreased, whereas the expression level of WNT5A was significantly increased. The activity of the Wnt/ $\beta$-catenin signaling pathway was upregulated; the level of LDH released was significantly increased; and activities of SOD and GSH-PX were significantly decreased. The aforementioned changes resulted in decreased cell activity and increased apoptosis rate. The overexpression of miR-26a-5p could reduce the expression level of WNT5A, the activity of the Wnt/ $\beta$-catenin signaling pathway, and the apoptosis rate and restore the cell viability.

These results suggest that miR-26a-5p can target WNT5A and thus, inhibit the Wnt/ $\beta$-catenin signaling pathway activity, inhibiting H/R-induced cardiomyocyte injury and apoptosis.
\end{abstract}

Key words: Acute myocardial infarction, SOD, GSH-PX, Apoptosis

(Int Heart J Advance Publication)

$\mathrm{C}$ ardiovascular disease is an increasingly common disease resulting from physical inactivity and unhealthy dietary habits, threatening public health and causing economic burden to the development of individuals and society. ${ }^{1)}$ Cardiac ischemia, whether caused by ischemic heart disease or atherosclerosis, can cause damage to cardiomyocytes. ${ }^{2)}$

Mammalian cardiomyocytes are defined as terminally differentiated cells that have a weak proliferation ability and almost no cell proliferation. ${ }^{3)}$ Therefore, once a cardiomyocyte is injured because of ischemia or any other factor, continuous ischemia-hypoxic myocardial necrosis would occur; apoptotic or programmed-death cardiomyocytes are replaced by fibroblasts, and myocardial remodeling is eventually induced, leading to ventricular dysfunction or even death. ${ }^{4}$ Clinical treatment of myocardial ischemia mainly adopts the strategy of reperfusion to restore the blood supply to myocardium, including percutaneous coronary intervention and coronary artery bypass grafting. ${ }^{5)}$ However, in the case of ischemic myocardial tissue injury, this restored blood supply would be absorbed by cardiomyocytes, causing further damage. This is known as myocardial hypoxia/reoxygenation (H/R) injury. $\left.{ }^{6}\right)$ Additionally, the main cause of acute myocardial infarction is the irreversible heart damage resulting from either myocardial hypoxia/reoxygenation (H/R) injury or cardiomyocytes apoptosis. ${ }^{7)}$ At present, restoring cardiac blood supply is an important treatment of cardiovascular diseases. However, current studies cannot provide better solutions to avoid myocardial cell injury caused by H/R. Therefore, it is particularly important to elucidate the molecular mechanism of myocardial cell injury caused by

From the ${ }^{1}$ Department of Ultrasound, Zhongshan Hospital Xiamen University, Xiamen, China, ${ }^{2}$ Department of Medicine, Fujian Medical University, Xiamen, China and ${ }^{3}$ Center of Clinical Laboratory, Zhongshan Hospital Xiamen University, Xiamen, China.

*These authors contribute equally to this work

This work was supported by the Joint Funds of Science and Technology Commission of Fujian Province of China (No. 2018J01396), the Youth Foundation Project of Fujian Provincial Health Department (No. 2014-2-69), the Natural Science Foundation of Fujian Province (No. 2015J01531), Overseas Students in Science and Technology Activities, Ministry of Human Resources and Social Security of the People's Republic of China (No. 2015), and Fujian Health and Family Planning Commission for Young and Middle-aged Talents Training Project (No. 2016-ZQN-87).

Address for correspondence: Yang Zhang, MD, The Center of Clinical Laboratory, Zhongshan Hospital Xiamen University, HuBin South Road No.201-205, Xiamen, Fujian Province, 361004, China. E-mail: yangzhang77@126.com

Received for publication January 25, 2021. Revised and accepted May 31, 2021

Released in advance online on J-STAGE September 17, 2021.

doi: 10.1536/ihj.21-054

All rights reserved by the International Heart Journal Association. 
$\mathrm{H} / \mathrm{R}$ and to provide further insights for clinical treatment.

MicroRNAs (miRNAs) are endogenous non-coding small RNA molecules with approximately 21-24 nucleotides. MiRNAs can induce effective gene silencing by complementary pairing with the 3'-untranslated region (3'-UTR) of target mRNAs. ${ }^{8,9)}$ Recent studies have demonstrated that miRNAs can control the transcriptional translation of approximately $60 \%$ of proteins, showing that miRNAs are widely involved in the signaling regulation of biological processes. ${ }^{10)}$ It has been confirmed that miRNAs can be involved in cellular activities including cell growth, apoptosis, and differentiation. ${ }^{11)}$ Also, miRNAs play a crucial role in the development of myocardial diseases, including H/R leading to cardiomyocyte injury. Wang, et al. pointed out that an up-regulation of miR-146a inhibited the expression of IRAK1 and TRAF6 and the activity of caspase-3/7/8 protein in cardiomyocytes, thereby alleviating cardiomyocyte injury induced by $\mathrm{H} / \mathrm{R}^{1{ }^{2}}{ }^{2}$ Zhai, et al. pointed out that miR-1 directly targets $\mathrm{Bcl}-2$ and prevents H/R-induced cardiomyocyte apoptosis. ${ }^{13)}$ In addition, miRNAs are also involved in the process of inducing cell injury. For example, Liu, et al. pointed out that the overexpression of miR-15b promotes apoptosis and loss of mitochondrial membrane potential, which in turn enhances H/R-induced cardiomyocyte apoptosis. ${ }^{14)} \mathrm{Gao}$, et al. suggested that miR-195 reduces the mRNA and protein expression of Bcl-2, Bax, and Cyt-c, thereby increasing H/R-induced myocardial injury. ${ }^{15)}$ It can be seen that miRNAs play different roles in biological information regulation during $\mathrm{H} / \mathrm{R}$ development to cardiomyocyte injury. However, previous studies have failed to clearly understand the mechanism of miRNA regulation of signaling pathways. Although it has been confirmed that miR-26a$5 p$ is involved in the regulation of the apoptosis process, ${ }^{16,17)}$ previous studies have only tried to elucidate the involvement of miR-26a-5p in the regulation of cardiomyocyte apoptosis in vivo, which does not fully illustrate the problem. Therefore, in this study, we establish an H/R injury model in vitro to reveal the specific relationship between miR-26a-5p and injury caused by $H / R$, as well as their related mechanism, which will make a contribution to an in-depth understanding of the injury mechanism of H/R.

\section{Methods}

Cell culture and treatment: $\mathrm{H} 9 \mathrm{C} 2$ cells were purchased from American Type Culture Collection (ATCC, USA). Cells were cultured under normal conditions in DMEM (Gibco, USA) with $10 \%$ fetal bovine serum (Gibco) and maintained in a $37^{\circ} \mathrm{C}$ incubator with $5 \% \mathrm{CO}_{2}$. $\mathrm{H} 9 \mathrm{C} 2$ cells were mainly divided into groups: 1) Control, 2) $\mathrm{NC}$ mimic, 3) miR-26a-5p: cells transfected with miR-26a-5p mimics, 4) NC inhibitor, 5) miR-26a-5p inhibitor: cells transfected with a miR-26a-5p inhibitor, and 6) H/R: H9C2 cells cultured in anaerobic and glucose-free DMEM under hypoxic conditions $\left(95 \% \quad \mathrm{~N}_{2}\right.$ and $\left.5 \% \mathrm{CO}_{2}\right)$ for 6 hours. After inducing hypoxia, H9C2 cells were cultured in DMEM under normoxic conditions (95\% air and 5\% $\mathrm{CO}_{2}$ ) for 12 hours; 7) H/R + NC and 8) H/R + miR-26a5 p or WNT5A: H9c2 cells were transfected with miR-26 a-5p mimics or pcDNA3.1-WNT5A followed by $\mathrm{H} / \mathrm{R}$ treatment; 9) $\mathrm{H} / \mathrm{R}+$ miR-26a-5p + WNT5A: H9c2 cells were transfected with miR-26a-5p mimics and pcDNA3.1WNT5A followed by H/R treatment. The cells were transfected according to Lipofectamine2000 (Invitrogen, USA) reagent instructions.

qRT-PCR assay: Total RNA was extracted from the cells with TRIzol (Invitrogen, USA), and cDNA was prepared according to the instructions of the random primer reverse transcription kit (Thermo, USA). The expression levels of each mRNA were tested using qRT-PCR following the instructions of the SYBRGREEN kit (TaKaRa, Japan), using GAPDH as an internal reference control, and six replicates were set up for the experiment. Experimental data were used to calculate the relative expression of the target gene using the $2^{-\Delta \Delta \mathrm{Ct}}$ method.

CCK-8 assay: $5 \times 10^{4}$ H9C2-treated cells were seeded into light-tight 96-well plates and supplemented with 200 $\mu \mathrm{L}$ of fresh or glucose-free DMEM complete medium for 24 hours. The cells were transfected and incubated for 24 hours. The cells were treated with $\mathrm{H} / \mathrm{R}$, and after 24 hours, 48 hours, and 72 hours of continuous culture, 10 $\mu \mathrm{L}$ CCK8 (Beyotime, China) was added to each well and placed in an incubator for 1.5 hours. The absorbance value (optical density, OD) at $450 \mathrm{~nm}$ was measured with a microplate reader; the absorbance value was collected; and the cell proliferation rate was calculated.

Flow cytometry: Trypsin was used to digest the cells of each group after intervention into a centrifuge tube; samples were centrifuged at $4^{\circ} \mathrm{C}, 800 \mathrm{rpm}$ for 5 minutes; and the cells were rinsed twice with precooled sterile PBS and centrifuged for 5 minutes each time. The cell concentration was adjusted to $5 \times 10^{5}$ cells $/ \mathrm{mL}$ with PBS after being centrifuged at $4{ }^{\circ} \mathrm{C}, 800 \mathrm{rpm}$ for 5 minutes. To $200 \mu \mathrm{L}$ of cell suspension, $10 \mu \mathrm{L}$ of AnnexinV-FITC and $10 \mu \mathrm{L}$ of the PI solution $(20 \mathrm{mg} / \mathrm{L})$ were added; then, the mixture was incubated at room temperature in the dark for 10 minutes. Finally, $500 \mu \mathrm{L}$ of PBS was added, and apoptosis was then detected using flow cytometry.

LDH concentration and measurement of SOD and GSH-PX activity: The culture supernatant of each group after treatment was collected. LDH concentration and SOD activity were detected using LDH and an SOD assay kit (A005-1-2, Nanjing Jiancheng Institute of Biological Engineering, China) according to the manufacturer's protocol, respectively. At the end of the reaction, the OD was measured at $450 \mathrm{~nm}$ using a microplate reader (Thermo, USA).

GSH-PX was performed by the GSH-PX kit (A0013-2, Nanjing Jiancheng Institute of Biological Engineering, China) according to the manufacturer's protocol. At the end of the reaction, GSH-PX activity was measured at $412 \mathrm{~nm}$ using a microplate reader.

Dual-luciferase reporter assay: The sequence of the binding site of miR-26a-5p and WNT5A and its mutant sequence were inserted into the downstream of the firefly luciferase gene to construct an expression vector (Promega, USA). The recombinant plasmids of miR-26a$5 \mathrm{p}$ mimics and pmirGLO-WNT5A-WT/MUT and the wild-type or mutant recombinant plasmids of miR-26a-5p $\mathrm{NC}$ and WNT5A in the control group were mixed with 
A

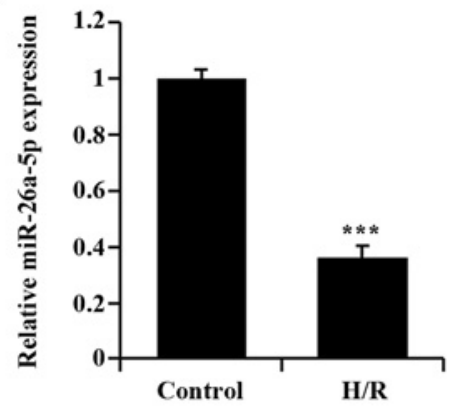

B

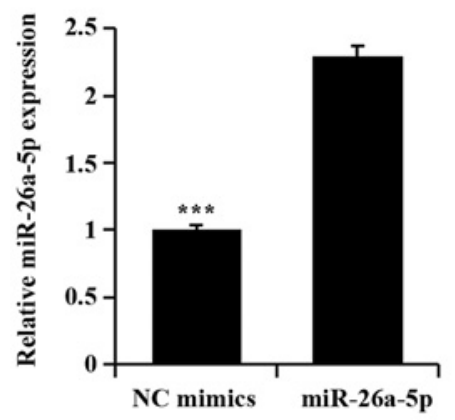

C

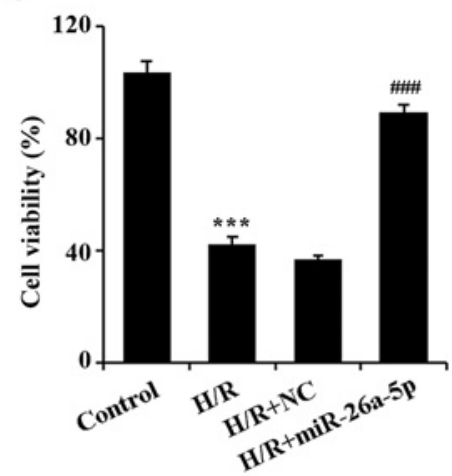

D

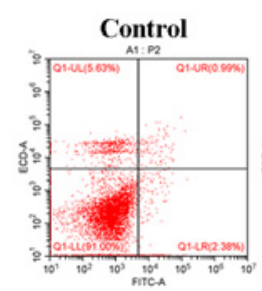

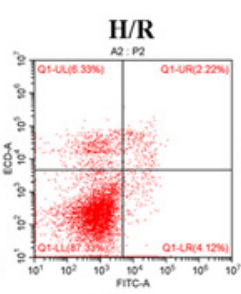
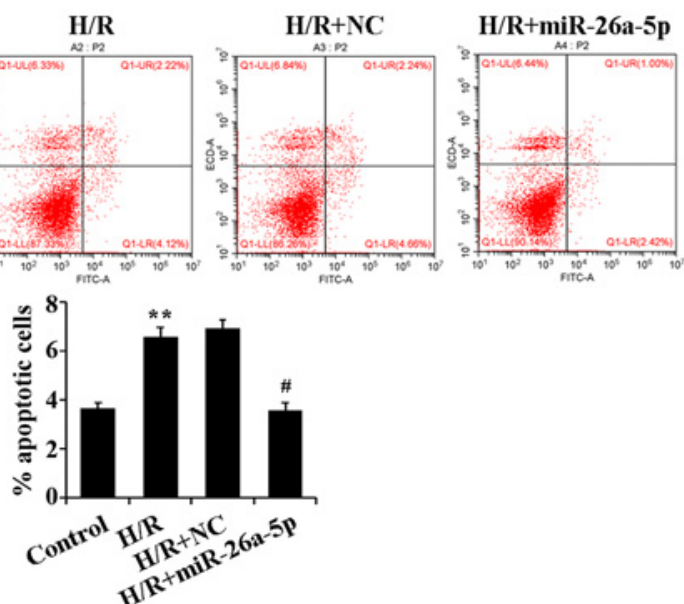

$\mathbf{E}$

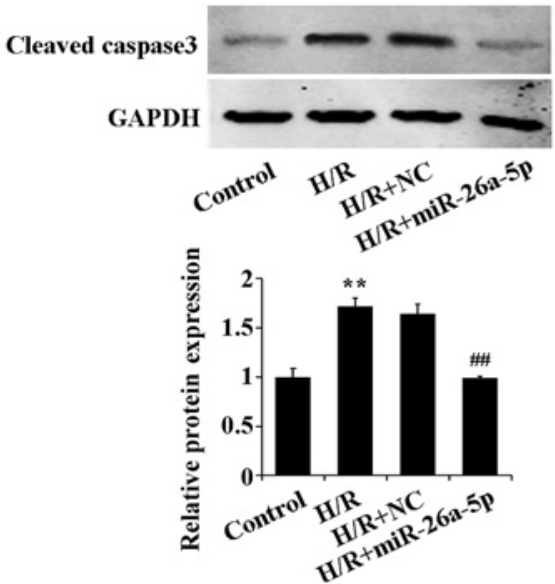

Figure 1. Effect of miR-26a-5p on H/R-induced proliferation and apoptosis of cardiomyocytes. A, B: The expression of miR-26a-5p in cells treated differently. C: CCK-8 to detect the cell viability of cells treated differently. D: Flow cytometry to detect the apoptosis of cells treated differently. E: The expression of cleave caspase 3 in cells treated differently. ${ }^{* *} P<0.01$ versus control group or NC group; ${ }^{* * *} P<0.001$. ${ }^{\#} P<0.05$ versus $\mathrm{H} / \mathrm{R}+\mathrm{NC}$ group; ${ }^{\#} P<0.01 ;{ }^{\# \#} P<0.001$. Each step of the experiment was repeated at least three times independently.

Lipo2000 liposomes and transfected into H9c2 cells. Forty-eight hours after transfection, luciferase activity was detected according to the double luciferase reporter gene detection kit.

Western blot: Proteins were extracted from treated cells using a RIPA lysis buffer. Then, the protein concentration was determined with BCA kit (Beyotime). Twenty micrograms of protein was denatured in a boiling bath with a loading buffer, separated by $10 \%$ SDS-PAGE, and then transferred to PVDF membranes. After being blocked by $5 \%$ nonfat milk for 1 hour at room temperature, the membrane was incubated with primary antibodies: cleaved caspase3 (ab2302, Abcam, Cambridge, UK), $\beta$-catenin (ab223075, Abcam), Cyclin D1 (ab16663, Abcam), c-myc (ab32072, Abcam), and WNT5A (ab17982, Abcam) at $4^{\circ} \mathrm{C}$ overnight. After the membranes were washed three times, secondary antibodies were added and incubated for 1 hour at room temperature. After another three washes with PBS, the membrane was visualized using a chemiluminescence reagent. Relative protein expression was calculated using GAPDH as an internal reference.

Statistical analysis: Each experiment was repeated three times independently. The measurement data are expressed as mean \pm standard deviation (SD). One-way analysis of variance was used to evaluate differences between more than two groups. Student's $t$-test was used for comparison between the two groups. SPSS 22.0 software was used for data analysis. A $P$-value of less than 0.05 was considered statistically significant.

\section{Results}

miR-26a-5p inhibited H/R-induced cardiomyocyte apoptosis: Compared with that in the control group, the expression level of miR-26a-5p was significantly reduced in the cells of the $\mathrm{H} / \mathrm{R}$ group (Figure $1 \mathrm{~A}, P<0.05$ ). Compared with the NC mimics group, the expression level of miR-26a-5p was significantly increased in the cells of the miR-26a-5p group (Figure 1B, $P<0.05$ ). Compared with that in the control group, the viability of cells in the H/R group was significantly decreased, and the apoptotic rate was significantly increased; compared with that in the $\mathrm{H} / \mathrm{R}+\mathrm{NC}$ group, the viability of cells in the $H / R+$ miR-26a-5p group was significantly increased, 
A

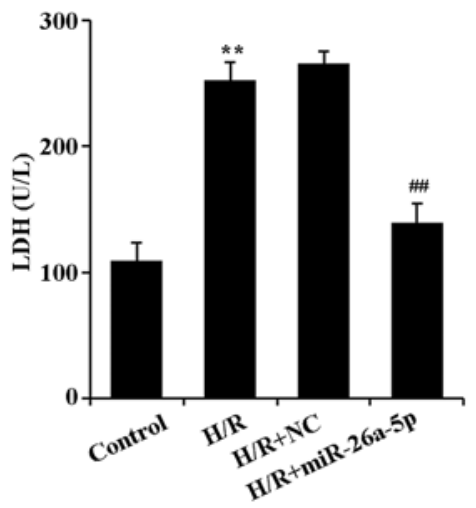

B

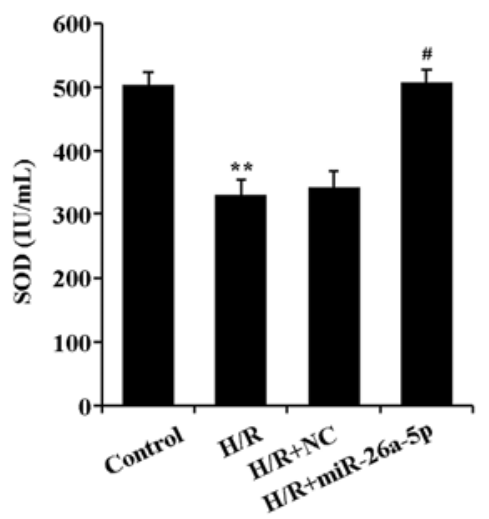

C

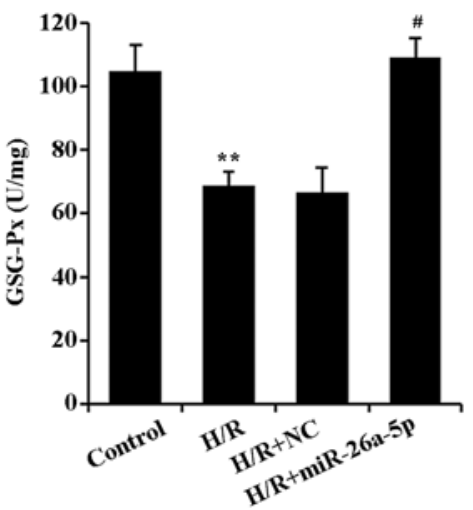

Figure 2. miR-26a-5p inhibited H/R-induced oxidative stress in cardiomyocytes. A: LDH release from cells with different treatments. B: SOD activity in cells with different treatments. C: GSH-PX activity in cells with different treatments. $* * P<0.01$ versus control group. ${ }^{\#} P<0.05$ versus $\mathrm{H} / \mathrm{R}+\mathrm{NC}$ group. ${ }^{\#} P<0.01$. Each step of the experiment was repeated at least three times independently.
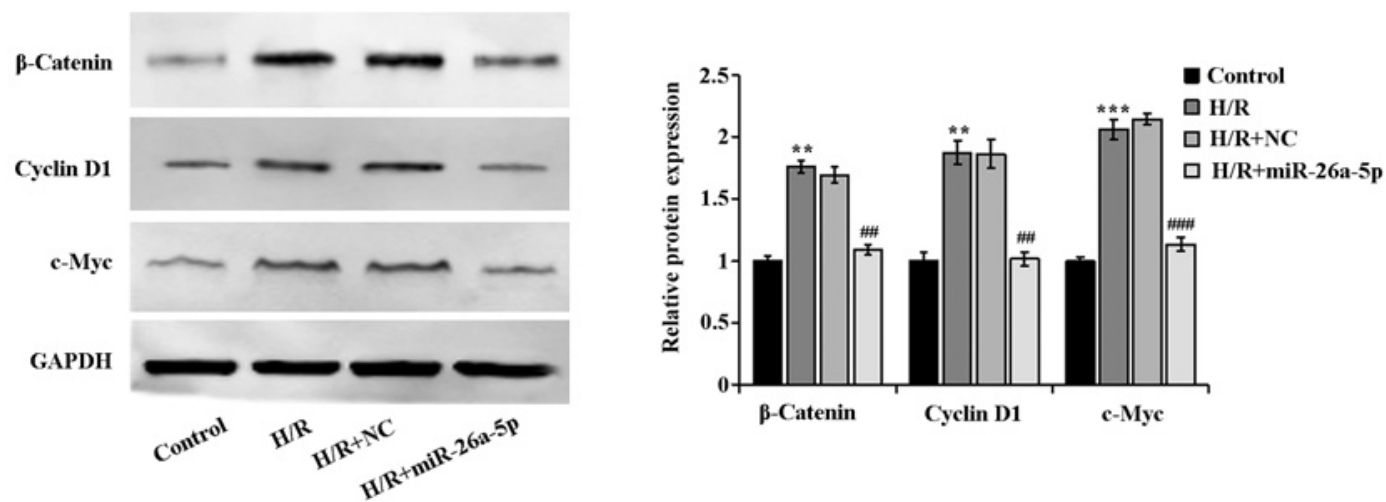

Figure 3. Effect of miR-26a-5p on the $\mathrm{Wnt} / \beta$-catenin signaling pathway in $\mathrm{H} / \mathrm{R}$-induced cardiomyocytes. $* * P<0.01$ versus control group. ${ }^{* * *} P<0.001$. ${ }^{\#} P<0.01$ versus $\mathrm{H} / \mathrm{R}+\mathrm{NC}$ group. ${ }^{\# \# \#} P<0.001$. Each step of the experiment was repeated at least three times independently.

and the apoptotic rate was significantly decreased (Figure $1 \mathrm{C}, \mathrm{D}, P<0.05)$. Compared with that in the control group, the expression level of cleaved caspase-3, a key protein of apoptosis, was significantly increased in the cells of the H/R group; compared with that in the $H / R+$ $\mathrm{NC}$ group, the expression level of cleaved caspase 3 was significantly decreased in the cells of the H/R + miR-26a$5 \mathrm{p}$ group (Figure $1 \mathrm{E}, P<0.05$ ). There was no significant difference between $\mathrm{H} / \mathrm{R}$ and $\mathrm{H} / \mathrm{R}+\mathrm{NC}$. These results indicate that in H/R-treated cardiomyocytes, high miR-26a-5 $\mathrm{p}$ expression is able to promote proliferation, inhibit apoptosis, and the expression of cleaved caspase- 3 protein in the cells, thereby reducing $\mathrm{H} / \mathrm{R}$-induced cardiomyocyte injury.

miR-26a-5p inhibited H/R-induced oxidative stress in cardiomyocytes: The results of biochemical tests revealed (Figure 2A-C) that compared with those in the control group, the release of LDH was significantly increased, and the activities of SOD and GSH-PX were significantly decreased in $\mathrm{H} / \mathrm{R}$; compared with that in the $\mathrm{H} / \mathrm{R}+\mathrm{NC}$ group, the level of LDH released was significantly de- creased, and the activities of SOD and GSH-PX were significantly increased in $\mathrm{H} / \mathrm{R}+\operatorname{miR}-26 \mathrm{a}-5 \mathrm{p}(P<0.05)$. These results confirm that the overexpression of miR-26a$5 p$ could inhibit $\mathrm{H} / \mathrm{R}$-induced oxidative stress response in cardiomyocytes.

Effect of miR-26a-5p on the Wnt/ $\beta$-catenin signaling pathway in H/R-induced cardiomyocytes: The activity of the Wnt/ $\beta$-catenin signaling pathway was determined by detecting the expressions of important signaling molecules: $\beta$-catenin, CyclinD1, and c-myc. As illustrated in Figure 3 , compared with those in the control group, the expression levels of $\beta$-catenin, CyclinD1, and c-myc protein were significantly increased in the H/R group; compared with those in the $\mathrm{H} / \mathrm{R}+\mathrm{NC}$ group, the expression levels of $\beta$-catenin, CyclinD1, and c-myc protein were significantly decreased in the $\mathrm{H} / \mathrm{R}+\mathrm{miR}-26 \mathrm{a}-5 \mathrm{p}$ group. It can be seen that the $\mathrm{Wnt} / \beta$-catenin signaling pathway could be activated in $H / R$ injury, whereas the upregulation of miR-26a-5p expression level is able to inhibit this signaling pathway.

Targeted binding of miR-26a-5p to WNT5A: After in- 
A

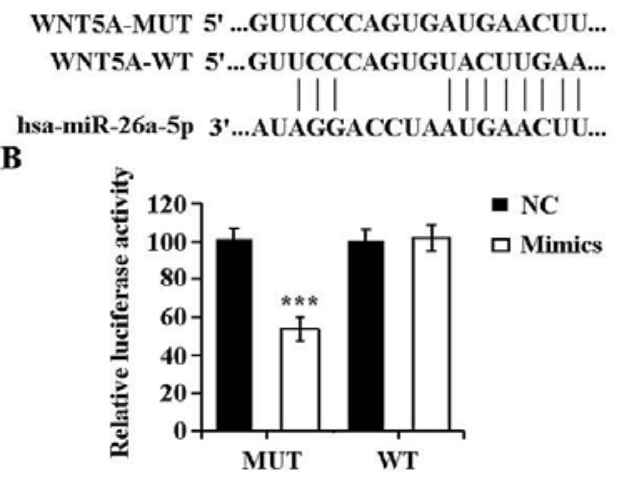

C

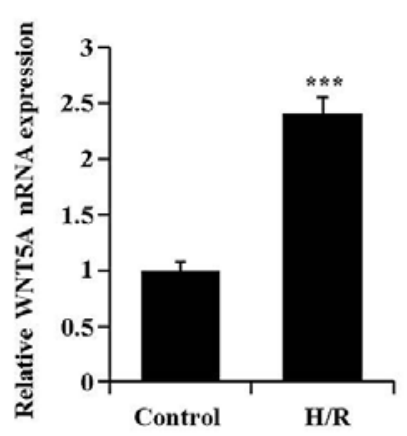

D

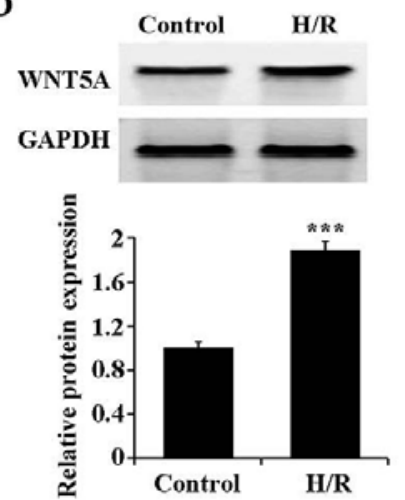

$\mathbf{E}$

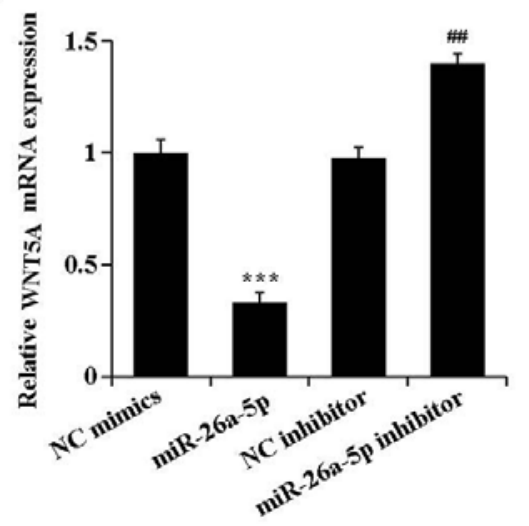

$\mathbf{F}$

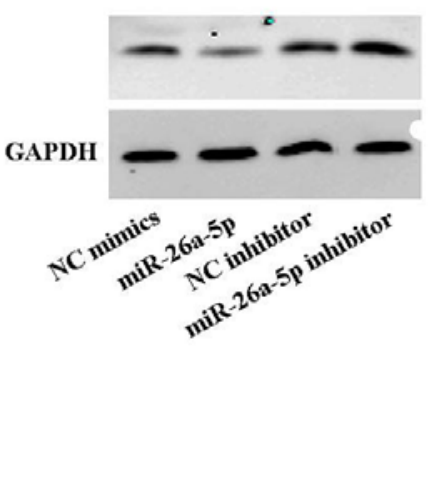

G

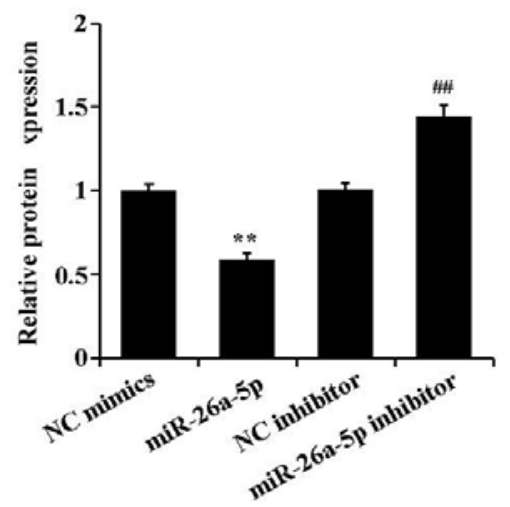

Figure 4. miR-26a-5p targets WNT5A. A: Schematic diagram of the interaction sequence between miR-26a-5p and WNT5A. B: Statistical diagram of fluorescence value of miR-26a-5p-targeted binding to WNT5A validated by dual-luciferase reporter assay. $* * * P<0.001$ versus NC group. C: mRNA expression level of WNT5A in cells with different treatments. $* * * P<0.001$ versus control group. D: Protein expression level of WNT5A in cells with different treatments. $* * * P<0.001$ versus control group. E: mRNA expression level of WNT5A in cells with different treatments. F, G: Protein expression level of WNT5A in cells with different treatments. $* * P<0.01$. $* * * P<0.001$ versus NC mimics group. ${ }^{\#} P<$ 0.01 versus NC inhibitor group. Each step of the experiment was repeated at least three times independently.

vestigating the link between miR-26a-5p and the $\mathrm{Wnt} / \beta$ catenin signaling pathway, miR-26a-5p was predicted to target and bind to WNT5A (Figure 4A). The results of the dual-luciferase reporter assay suggested an interaction between miR-26a-5p and WNT5A (Figure 4B). Compared with those in the control group, the mRNA and protein expression levels of WNT5A were significantly increased in the H/R cells (Figure 4C, D, $P<0.05$ ). Compared with those in the NC mimics group, the mRNA and protein expression levels of WNT5A in the miR-26a-5p group were significantly decreased; compared with those in the $\mathrm{NC}$ inhibitor group, the expression levels of WNT5A in the miR-26a-5p inhibitor group were significantly increased (Figure 4E-G, $P<0.05$ ). The above results suggest that miR-26a-5p was able to regulate the $\mathrm{Wnt} / \beta$-catenin signaling pathway by targeting and binding to WNT5A.

MiR-26a-5p reversed the effects of WNT5A on H/Rinduced cardiomyocytes: Compared with those in the H/ $R$ group, the viability of cells in the H/R + WNT5A group was significantly decreased, and the apoptotic rate was significantly increased; compared with those in the $\mathrm{H} /$ $\mathrm{R}+$ WNT5A group, the viability of cells in the H/R + miR-26a-5p + WNT5A group was significantly increased, and the apoptotic rate was significantly decreased (Figure 5A, B, $P<0.05)$. Compared with that in the H/R group, the expression level of cleaved caspase 3 was significantly increased in the cells of the H/R + WNT5A group; compared with that in the H/R + WNT5A group, the expression level of cleaved caspase 3 was significantly decreased in the cells of the $H / R+$ miR-26a-5p + WNT5A group (Figure 5C, $P<0.05$ ).

\section{Discussion}

Recently, studies have pointed out that miRNAs are associated with H/R injury-induced cardiomyocyte apoptosis and regulation of cardiac function. ${ }^{18)}$ Some miRNAs were found to be dysregulated in cardiomyocytes that initiate apoptosis as a result of $\mathrm{H} / \mathrm{R}$ injury, such as miR$21,{ }^{19)}$ miR-29, ${ }^{20)}$ and miR-214. ${ }^{21)}$ Although some studies have pointed out that these dysregulated miRNAs are related to cell proliferation, apoptosis, or programmed cell death, their biological functions and mechanisms remain elusive. Like most miRNAs, miR-26a-5p has diverse biological functions and is involved in apoptosis and metastasis of cells. ${ }^{22}$ It has been stated that the up-regulation of 
A

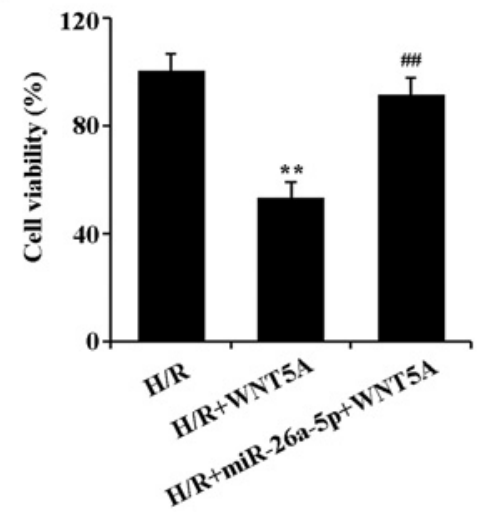

B

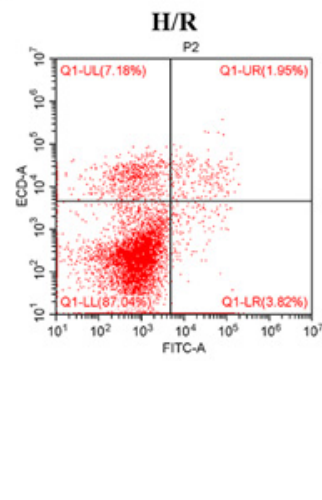

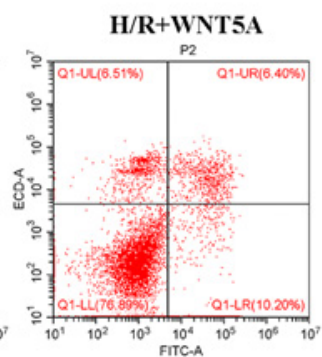

H/R+miR-26a-5p+WNT5A
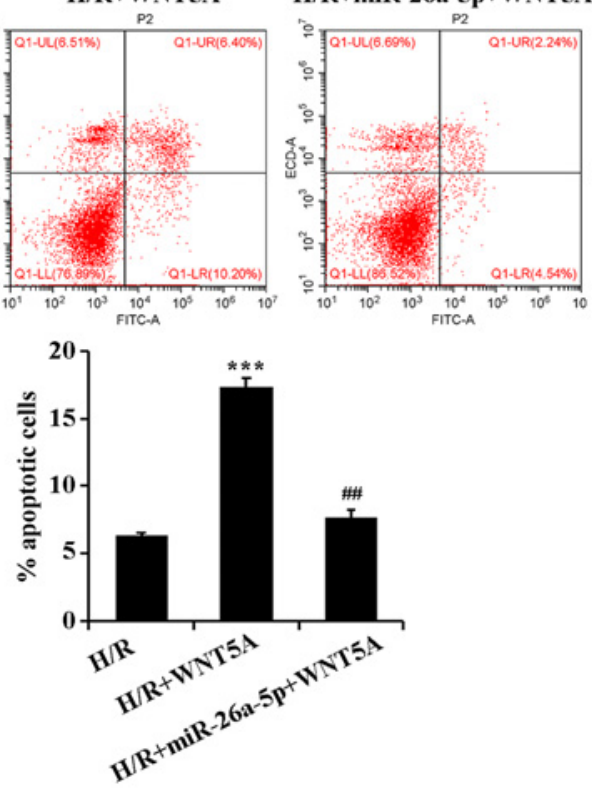

C
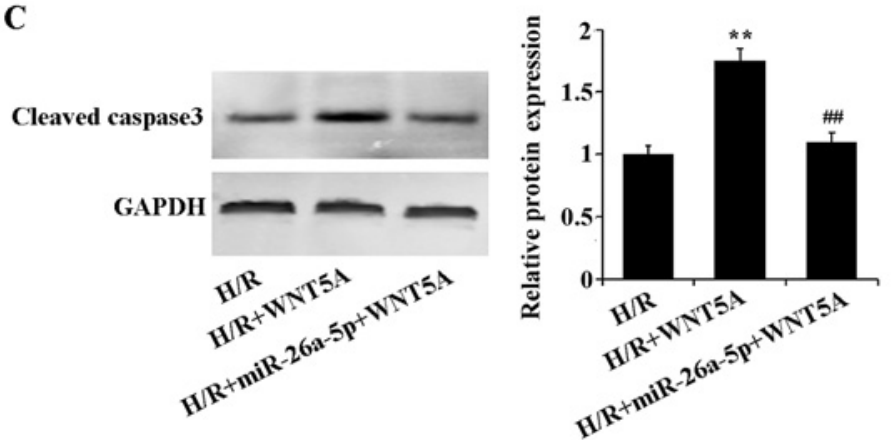

Figure 5. miR-26a-5p reversed the effect of WNT5A on H/R-induced cardiomyocytes. A: CCK-8 was used to detect the cell viability of cells treated differently. B: Flow cytometry was used to detect the apoptosis of cells treated differently. C: The expression of cleaved caspase 3 in cells treated differently. ${ }^{* *} P<0.01$ versus $\mathrm{H} / \mathrm{R}$ group. ${ }^{* * *} P<0.001$. ${ }^{\# \#} P<0.01$ versus $\mathrm{H} / \mathrm{R}+\mathrm{WNT}$ AA group. Each step of the experiment was repeated at least three times independently.

miR-26a-5p improves cardiac function and inhibits myocardial inflammation. ${ }^{23)}$ In addition, miR-26a-5p can inhibit autophagy in cardiac fibroblasts by targeting ULK1, which in turn can avoid cardiac hypertrophy and heart failure. ${ }^{24}$ Based on the key role of miR-26a-5p in the development of cardiovascular disease, as presented in previous studies, this study further showed that miR-26a-5p has an important role in $\mathrm{H} / \mathrm{R}$ injury of cardiomyocytes and verified the inhibitory effect of miR-26a-5p on the $\mathrm{Wnt} / \beta$-catenin signaling pathway activity by targeting and binding to WNT5A.

According to the experimental results of this study, the expression of miR-26a-5p in cardiomyocytes after H/R injury was significantly reduced. In addition, the overexpression of miR-26a-5p could significantly increase the cell viability of cardiomyocytes after $H / R$ injury, reduce the expression of cleaved caspase 3 protein, and inhibit apoptosis. Thus, miR-26a-5p was demonstrated to protect cardiomyocytes from $\mathrm{H} / \mathrm{R}$ damage. A previous study demonstrated that miR-26a-5p was significantly downregulated in rats with diabetic myocardial injury; miR26a-5p could target and suppress downstream factors and inhibit diabetes-induced cardiomyocyte apoptosis, thereby reducing myocardial injury. ${ }^{25)}$ In addition, miR-26a-5p can affect the proliferation, invasion, and apoptosis of fibroblast-like synoviocytes by controlling the PI3K/AKT signaling pathway. ${ }^{26)}$ Thus, it is speculated that miR-26a-
$5 p$ may have a regulatory effect on the viability and apoptosis of cells in vivo overall. Meanwhile, the experimental results in this study indicated that the release of $\mathrm{LDH}$ was significantly increased and the activities of SOD and GSH-PX were significantly decreased in H/R injury; however, the up-regulation of the miR-26a-5p expression level could significantly reduce the release of LDH and significantly increase activities of SOD and GSH-PX, indicating that miR-26a-5p overexpression could also inhibit the oxidative stress response of cardiomyocytes during $H / R$.

At present, miR-26a-5p has been demonstrated to regulate multiple genes and participate in the process of cancer development. ${ }^{22)}$ For example, miR-26a-5p promotes the metastasis of lung cancer cells by targeting ITG $\beta 8 .{ }^{26)}$ However, there is a lack of reported study on the mechanism of which miR-26a-5p targets and regulates the biological status of cardiomyocytes after H/R injury. Therefore, in this study, we used a bioinformatics website to first find the target gene of miR-26a-5p and then select a potential candidate, which was WNT5A. WNT5A is an important member of the Wnt family and is involved in activating a variety of non-canonical Wnt signaling pathways by binding to different members of Ror family receptors. ${ }^{27)}$ WNT5A signaling is essential for regulating normal developmental processes, including proliferation, differentiation, migration, adhesion, and polarity. ${ }^{28)}$ Among them, the Wnt/ $\beta$-catenin signaling pathway, in which 
WNT5A is involved, is a key pathway regulating apoptosis and viability. ${ }^{29)}$ According to the results of the dualluciferase reporter assay in this study, WNT5A expression was significantly up-regulated in the cardiomyocytes injured by $\mathrm{H} / \mathrm{R}$ and was negatively regulated by miR-26a$5 \mathrm{p}$. A previous study has been shown that by modulating the SFRP4/Wnt/ $\beta$-catenin signaling pathway, MeCP2 is able to reduce the damage caused by $H / R$ to cardiomyocytes. ${ }^{30)}$ In our study, Wnt/ $\beta$-catenin signaling pathway activity also changed alongside WNT5A expression variation. Thus, it is speculated that H/R decreases the expression of miR-26a-5p and up-regulates WNT5A to activate the $\mathrm{Wnt} / \beta$-catenin signaling pathway for the purpose of inhibiting the viability of cardiomyocytes and promoting their apoptosis.

\section{Conclusions}

In summary, during $H / R$ treatment of ischemic heart disease, down-regulated miR-26a-5p expression can promote Wnt/ $\beta$-catenin signaling pathway activity by increasing the expression of WNT5A, thereby inhibiting the cellular activity of cardiomyocytes, up-regulating cleaved caspase 3 protein and promoting cardiomyocyte apoptosis. This study suggests that miR-26a-5p can play a role in protecting cardiomyocytes during $\mathrm{H} / \mathrm{R}$ treatment. However, since this study is limited on a rat cell model, further experiments are required to fully clarify the protective effect of miR-26a-5p on cardiomyocytes.

\section{Disclosure}

Conflicts of interest: The authors declare that there is no conflict of interest between them.

Data availability statement: Data sharing not applicable-no new data generated.

\section{References}

1. Sun H, Gusdon AM, Qu S. Effects of melatonin on cardiovascular diseases: progress in the past year. Curr Opin Lipidol 2016; 27: 408-13.

2. Omura JD, Bellissimo MP, Watson KB, Loustalot F, Fulton JE Carlson SA. Primary care providers' physical activity counseling and referral practices and barriers for cardiovascular disease prevention. Prev Med 2018; 108: 115-22.

3. Zhao N, Mi L, Zhang X, et al. Enhanced MiR-711 transcription by PPAR $\gamma$ induces endoplasmic reticulum stress-mediated apoptosis targeting calnexin in rat cardiomyocytes after myocardial infarction. J Mol Cell Cardiol 2018; 118: 36-45.

4. Xu T, Ding W, Ji X, et al. Oxidative stress in cell death and cardiovascular diseases. Oxid Med Cell Longev 2019; 2019 9030563

5. Tang GY, Meng X, Li Y, Zhao CN, Liu Q, Li HB. Effects of vegetables on cardiovascular diseases and related mechanisms. Nutrients 2017; 9: 857.

6. Fan L, Zhou W, Zhang L, Jiang D, Zhao Q, Liu L. Sitagliptin protects against hypoxia/reoxygenation (H/R)-induced cardiac microvascular endothelial cell injury. Am J Transl Res 2019; 11 : 2099-107.

7. Guo Y, Luo F, Liu Q, Xu D. Regulatory non-coding RNAs in acute myocardial infarction. J Cell Mol Med 2017; 21: 1013-23.

8. Bartel DP. MicroRNAs: genomics, biogenesis, mechanism, and function. Cell 2004; 116: 281-97.

9. Lewis BP, Burge CB, Bartel DP. Conserved seed pairing, often flanked by adenosines, indicates that thousands of human genes are microRNA targets. Cell 2005; 120: 15-20.

10. Ambros V. The functions of animal microRNAs. Nature 2004; 431: 350-5.

11. Chen Y, Stallings RL. Differential patterns of microRNA expression in neuroblastoma are correlated with prognosis, differentiation, and apoptosis. Cancer Res 2007; 67: 976-83.

12. Wang X, Ha T, Liu L, et al. Increased expression of microRNA146 a decreases myocardial ischaemia/reperfusion injury. Cardiovasc Res 2013; 97: 432-42.

13. Zhai C, Tang G, Peng L, et al. Inhibition of microRNA-1 attenuates hypoxia/re-oxygenation-induced apoptosis of cardiomyocytes by directly targeting Bcl-2 but not GADD45Beta. Am J Transl Res 2015; 7: 1952-62.

14. Liu L, Zhang G, Liang Z, et al. MicroRNA-15b enhances hypoxia/reoxygenation-induced apoptosis of cardiomyocytes via a mitochondrial apoptotic pathway. Apoptosis 2014; 19: 19-29.

15. Gao CK, Liu H, Cui CJ, Liang ZG, Yao H, Tian Y. Roles of MicroRNA-195 in cardiomyocyte apoptosis induced by myocardial ischemia-reperfusion injury. J Genet 2016; 95: 99-108.

16. Huang Z, Xing S, Liu M, et al. MiR-26a-5p enhances cells proliferation, invasion, and apoptosis resistance of fibroblast-like synoviocytes in rheumatoid arthritis by regulating PTEN/PI3K/ AKT pathway. Biosci Rep 2019; 39.

17. Zhong X, Zhang L, Li Y, Li P, Li J, Cheng G. Kaempferol alleviates ox-LDL-induced apoptosis by up-regulation of miR-26a$5 p$ via inhibiting TLR4/NF- $\kappa B$ pathway in human endothelial cells. Biomed Pharmacother 2018; 108: 1783-9.

18. Di Y, Lei Y, Yu F, Changfeng F, Song W, Xuming M. MicroRNAs expression and function in cerebral ischemia reperfusion injury. J Mol Neurosci 2014; 53: 242-50.

19. Cheng Y, Zhu P, Yang J, et al. Ischaemic preconditioningregulated miR-21 protects heart against ischaemia/reperfusion injury via anti-apoptosis through its target PDCD4. Cardiovasc Res 2010; 87: 431-9.

20. Ye Y, Hu Z, Lin Y, Zhang C, Perez-Polo JR. Downregulation of microRNA-29 by antisense inhibitors and a PPAR-gamma agonist protects against myocardial ischaemia-reperfusion injury. Cardiovasc Res 2010; 87: 535-44.

21. Park KM, Teoh JP, Wang Y, et al. Carvedilol-responsive microRNAs, miR-199a-3p and -214 protect cardiomyocytes from simulated ischemia-reperfusion injury. Am J Physiol Heart Circ Physiol 2016; 311: H371-83.

22. Chang L, Li K, Guo T. miR-26a-5p suppresses tumor metastasis by regulating EMT and is associated with prognosis in HCC. Clin Transl Oncol 2017; 19: 695-703.

23. Kong B, Qin Z, Ye Z, Yang X, Li L, Su Q. microRNA-26a-5p affects myocardial injury induced by coronary microembolization by modulating HMGA1. J Cell Biochem 2019; 120: 10756-66.

24. Zheng L, Lin S, Lv C. MiR-26a-5p regulates cardiac fibroblasts collagen expression by targeting ULK1. Sci Rep 2018; 8: 2104.

25. Cai SS, Tao XW, Long Y, Xia K, Zhang Y. Effect of miR-26a on diabetic rats with myocardial injury by targeting PTEN. Eur Rev Med Pharmacol Sci 2020; 24: 10307.

26. Song Q, Liu B, Li X, et al. MiR-26a-5p potentiates metastasis of human lung cancer cells by regulating ITG $\beta 8$ - JAK2/STAT3 axis. Biochem Biophys Res Commun 2018; 501: 494-500.

27. Holland JD, Klaus A, Garratt AN, Birchmeier W. Wnt signaling in stem and cancer stem cells. Curr Opin Cell Biol 2013; 25: 254-64.

28. Chen CM, Orefice LL, Chiu SL, et al. Wnt5a is essential for hippocampal dendritic maintenance and spatial learning and memory in adult mice. Proc Natl Acad Sci U S A 2017; 114: E619-28.

29. van Amerongen R, Fuerer C, Mizutani M, Nusse R. Wnt5a can both activate and repress $\mathrm{Wnt} / \beta$-catenin signaling during mouse embryonic development. Dev Biol 2012; 369: 101-14. 
30. Li N, Zhang T, He M, Mu Y. MeCP2 attenuates cardiomyocyte hypoxia/reperfusion-induced injury via regulation of the SFRP4/
Wnt/ß-catenin axis. Biomarkers 2021; 26: 363-70. 